\title{
FATORES DESENCADEANTES DE CRANIOSSINOSTOSE: UM ESTUDO DA ARTE SOBRE O CONHECIDO E O NOVO PRESENTE NA LITERATURA
}

\author{
Matheus Henrique de Souza, Larissa Gasquez Magnesi, Janete Caprioli Carrocini \\ Universidade do Oeste Paulista - UNOESTE, Curso de Medicina, Presidente Prudente, SP. e-mail: janete@unoeste.br
}

\begin{abstract}
RESUMO
Craniossinostose (CS) é um distúrbio na qual uma ou mais suturas da calvaria se fundem prematuramente, o que limita o crescimento do osso local, acarretando em crânios anômalos. Este estudo tem como objetivo expor à população, através de citações e discussões, os fatores causais da CS, visando promover uma autonomia dos indivíduos, através de um maior conhecimento do assunto, pretendendo assim abrir caminhos para a elaboração de ações que objetivam diminuir a incidência desta comorbidade. Foi realizada uma revisão de artigos publicados somente em língua inglesa e foram associados à CS fatores genéticos, teratógenos, tabagismo durante gestação, idade parental avançada, níveis educacionais dos pais, uso de antidepressivos na gestação, estimulação pelos hormônios da tireóide, terapia com metotrexato, condições metabólicas e ingestão de certos nutrientes, expondo os mecanismos para a formação desta patogenia. 0 conhecimento dos fatores causais se torna fundamentais para a definição de estratégias visando diminuir a incidência de CS.
\end{abstract}

Palavras-chave: etiologia, craniossinostose, suturas, crânio, fisiopatologia.

\section{TRIGGERING CRANIOSYNOSTOSIS FACTORS: A STUDY OF ART ON THE KNOWN AND THE NEW PRESENT IN THE LITERATURE}

\begin{abstract}
Craniosynostosis (CS) is a disease in which one or more of the skull sutures to close early, which limits the growth of the bone site, resulting in anomalous skulls. This study aims to expose the population, through citations and discussions, the causal factors of CS, promoting an autonomy of the individuals, through greater knowledge of the subject, intending, thereby, open paths for the development of actions that aim decrease the incidence this comorbity. Was conducted a revision of articles published only in English and were found related with CS genetic factors, teratogen, smoking during pregnancy, advanced parental age, educational levels of parents, use of antidepressants in pregnancy, stimulation by thyroid hormones, with methotrexate therapy, metabolic conditions and intake of certain nutrients, showing the mechanisms for the formation this disease. The knowledge about the causal factors become important for the define of strategies that aim decrease the incidence of CS.
\end{abstract}

Keywords: etiology, craniosynostoses, sutures, skull, physiopathology

\section{INTRODUÇÃO}

Craniossinostose (CS) é um distúrbio na qual uma ou mais das cinco principais suturas da calvaria se fundem prematuramente, limitando assim o crescimento do osso local, na qual acarreta em crânios anômalos e assimétricos ${ }^{1}$, encontrando-se relatos de acometimento de sutura lambdóidea nos humanos desde o período Pleistoceno Médio².

A CS foi utilizada como termo para a fusão prematura das suturas cranianas pela primeira Colloq Vitae 2018 mai-ago; 10(2): 65-77. DOI: 10.5747/cv.2018.v10.n2.v234 ISSN 1984-6436/@ 2018 - Publicado pela Universidade do Oeste Paulista. Artigo Open Access sob uma licença CC BY-NC-ND (http://creativecommons.org/licenses/by-nc-nd/4.0/). vez por Otto, em $1830^{3}$. Em 1850, Virchow foi o primeiro a indicar que na fusão prematura da sutura da calota craniana ocorreria um crescimento compensatório em um plano paralelo à sutura fundida, com um crescimento mínimo em um plano perpendicular, além disso, ele propôs que a fusão prematura está associada com a inflamação das meninges ${ }^{4}$. Em 1935, Crouzon observou que poderia haver uma contribuição genética para a patogênese da $\mathrm{CS}^{5}$, e em 1959, Moss propôs que a CS resulta de um 
desenvolvimento anormal da base do crânio, levando a transmissão de forças mecânicas alterados à sutura sobrejacente. Paul Tessier, posteriormente, inaugurou a era moderna da cirurgia craniofacial com sua descrição da total remodelação da calota craniana para aumentar o volume intracraniano e criar uma aparência mais normal ${ }^{6}$.

A CS é relativamente comum na população em geral, ocorrendo em 1 a cada 2000-2500 nascidos ${ }^{7}$, podendo ser classificada de diversas formas: A CS pode ser dividida em não sindrômica ou sindrômica. Na primeira, não ocorrem outras manifestações concomitantemente com a CS que não sejam aquelas relacionadas com a fusão prematura das suturas ${ }^{8}$, enquanto que na segunda, existe a presença de outras deformidades que envolve diferentes sistemas do corpo, como cardíaco, geniturinário e musculoesquelético ${ }^{9}$. A CS pode ser classificada também através do número de pontos que se fundem prematuramente, como CS simples (uma sutura apenas) ou complexa (duas ou mais suturas) $^{10}$. Uma outra classificação possível é de acordo com a etiopatogenia da doença, na qual se for devido a um defeito intrínseco, é denominada CS primária, enquanto que as CS resultantes de outras condições médicas, como hipertireoidismo, talassemia, desordens metabólicas e hematológicas, são designadas como CS secundária ${ }^{8}$.

Fenótipos craniofacias de CS podem variar de normal até modificações da morfologia da abóbada craniana. Na maioria dos casos, as suturas estão fundidas prematuramente no nascimento, mas existem casos na qual os fenótipos da CS estão ausentes no nascimento e evoluem gradativamente durante os primeiros anos de vida ${ }^{11,12}$. Os fenótipos mais comuns são, na sinostose unilateral coronal ou lambdoidal, o crescimento antero-posterior, chamado de plagiocefalia (plágios, do grego "oblíquo"), sinostose coronal bilateral produz braquicefalia (brachys, do grego "a curto/amplo"), sinostose sagital resulta em escafocefalia (skaphe, do grego "em forma de barco") e sinostose metópica provoca trigonocefalia (trigonon, do grego "em forma de triângulo" $)^{10}$.

As suturas comumente afetadas na CS, classificando do mais para o menos frequente, são a sagital, coronal, metópica e a lambdóidea, ${ }^{9,13}$. A sagital ocorre em aproximadamente um em cada 5.000 nascimentos e constitui $40-55 \%$ dos casos não sindrômicos reconhecidos. Ocorre predominan-temente no sexo masculino, na qual a maioria dos casos possui $6 \%$ de hipótese de ser familiar (Figura $1)^{14,15}$
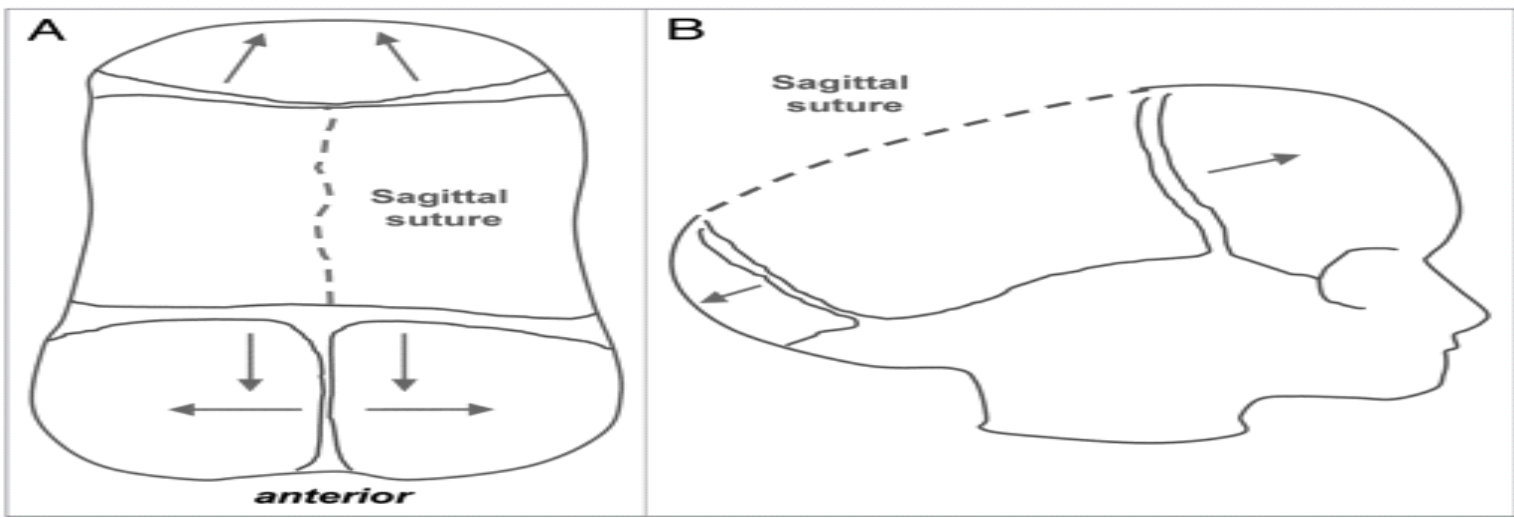

Figura 1. Representa uma Escafocelia por comprometimento da sutura sagital. Pode-se notar um crescimento ântero-posterior do crânio compensatório devido ao não crescimento correto latero-lateral, ocasionado pelo fechamento precoce da sutura. Modificada de Senarath-yapa et al. ${ }^{4}$

Sinostose coronal não sindrômica é responsável por $20-25 \%$ dos casos que ocorre em um a cada 10.000 nascimentos, podendo ocorrer de forma uni ou bilateralmente, acontecendo com uma maior frequência no sexo feminino e possuindo de $8-15 \%$ a chance de haver uma história familiar (Figura 2) $)^{1,15,16}$. 

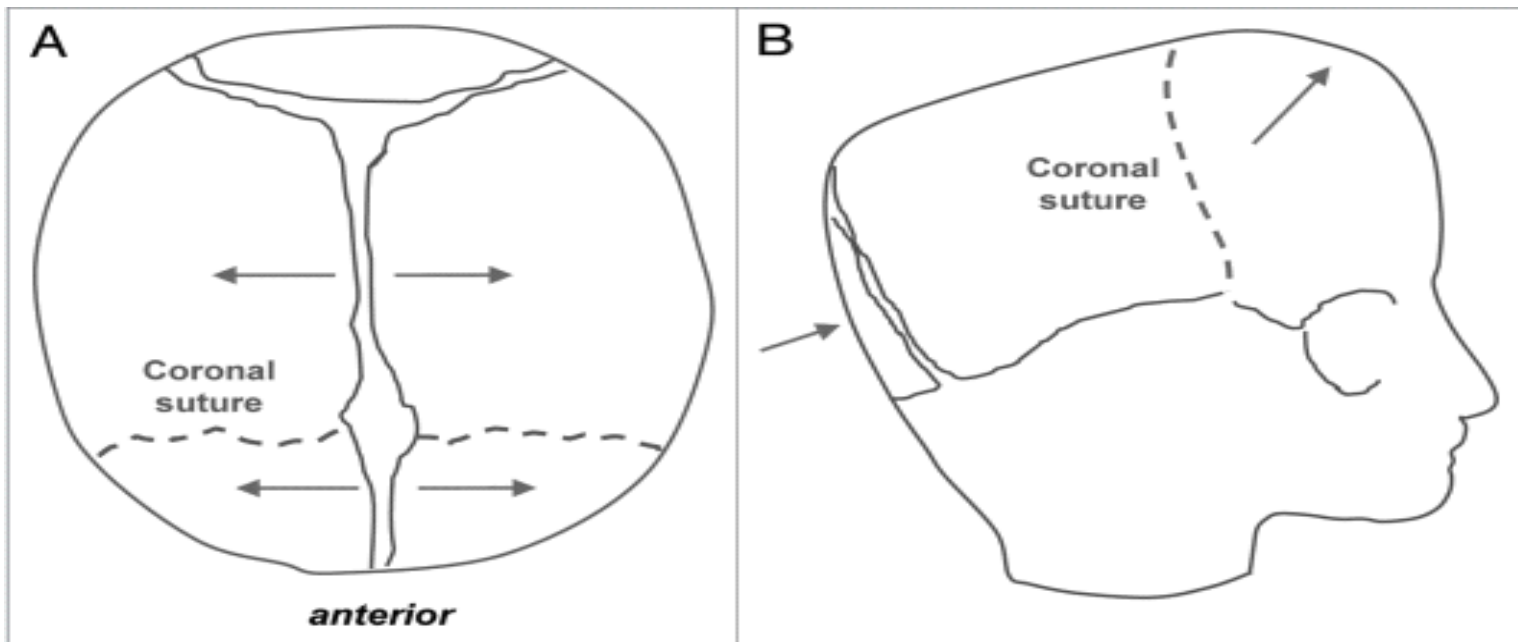

Figura 2. Uma Braquicefalia causada pela fusão prematura bilateral da sutura coronal. Nota-se um crescimento latero-lateral e anteriormente compensatório devido ao não crescimento posteriormente, ocasionando pelo fechamento precoce da sutura. Modificada de Senarath-yapa et al. ${ }^{4}$

A sinostose metópica é de aproximadamente um em cada 15.000 nascimentos ${ }^{13}$, é responsável por $14 \%$ dos casos não sindrômicos de CS, possuindo uma maior incidência em homens, apresentando geralmente $70 \%$ de não ocorrer outras malformações clínicas, e possuindo de 5$10 \%$ de chance de ter histórico familiar positiva (Figura 3) ${ }^{15,17,18}$.
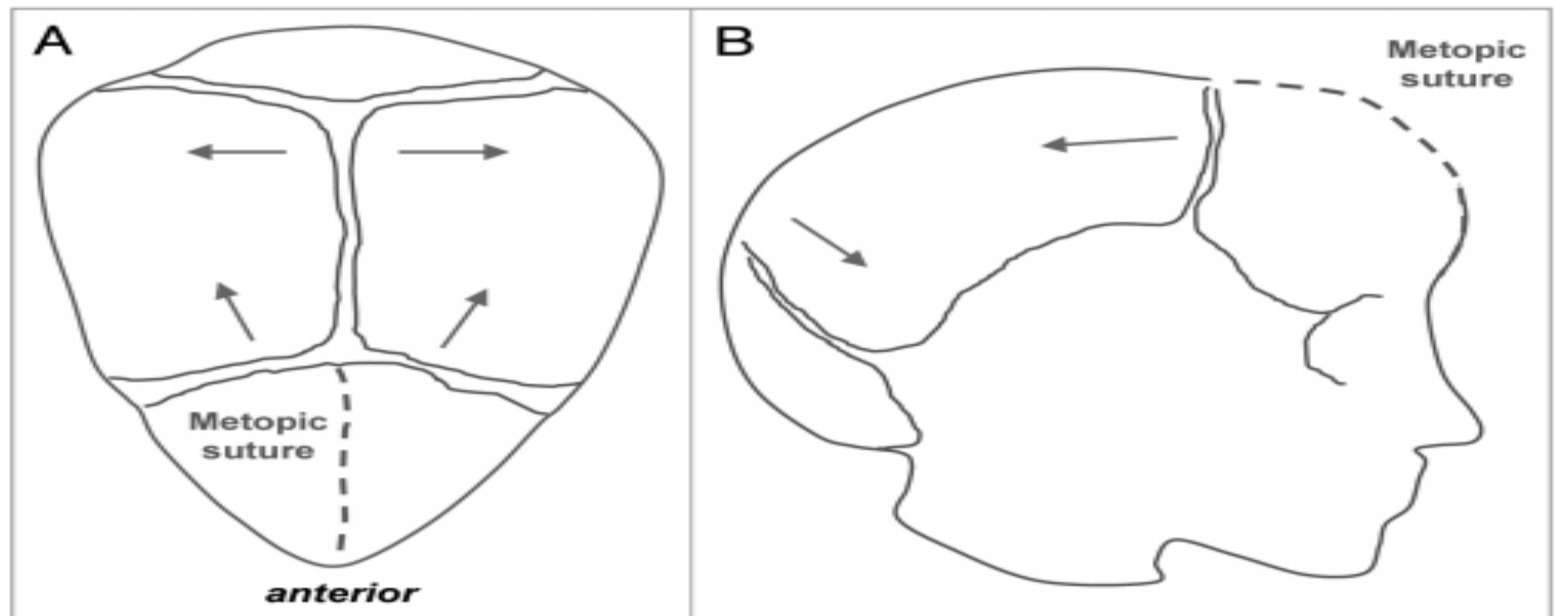

Figura 3. Uma trigonocefalia causada pela fusão prematura da sutura metópica. Nota-se um crescimento latero-posterior compensatório devido ao não crescimento latero-anterior, ocasionado pelo fechamento precoce da sutura craniana. Modificada de Senarath-yapa et al. ${ }^{4}$

As sinostoses lambdóideas apresentam 3-5\% das CS não sindrômicas ${ }^{15,19,20}$, ocorrem em 1 a cada 33.000 nascimentos $^{21}$, possuindo a genética envolvida em grande parte desconhecida e apresentando uma rara história familiar positiva (Figura 4) $)^{22}$. 


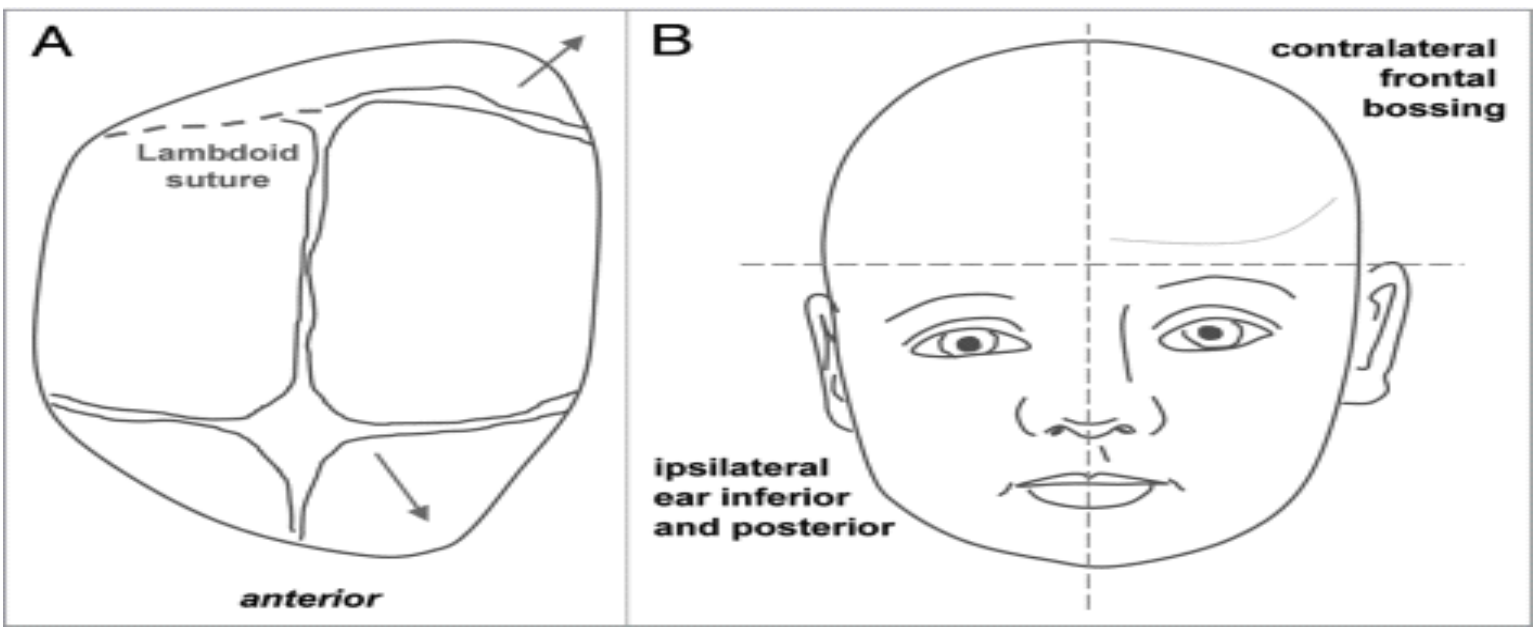

Figura 4. Uma plagiocefalia causada pela fusão prematura parcial da sutura lambdoide. Nota-se um crecimento compensatório para às diagonais devido ao não crescimento da sutura craniana. Modificada de Senarath-yapa et al. ${ }^{4}$

Os ossos da calvaria formam-se pela proliferação e diferenciação de células mesenquimais multipotentes em osteoblastos ${ }^{23}$, portanto, qualquer perturbação neste processo pode ser causador da fusão prematura das suturas cranianas ${ }^{24}$.

Esta revisão de literatura tem como objetivo expor à população em geral, através de citações e discussões, os fatores causais da CS, procurando promover uma autonomia dos mesmos, através de um maior conhecimento a respeito do assunto, visando também abrir um caminho para a elaboração de estratégias que objetivam diminuir a incidência da comorbidade apresentada.

\section{METODOLOGIA}

Realizou-se uma revisão integrativa da literatura através de artigos publicados em língua inglesa, encontrando os mesmos em bases de dados como PubMed, LILACS, Periódico Capes e Scielo a partir das palavras chaves "etiology, craniosynostoses, sutures, skull e physiopathology". Uma minuciosa leitura foi realizada na qual se procurou retirar dos artigos informações afirmativas, sem titubeio, sobre fatores que contribuam para o desenvolvimento da CS. Foram excluídos do estudo artigos em outros idiomas que não fosse o inglês e estudos cujas conclusões eram indefinidas.

\section{DESENCADEANTES DE CRANIOSSINOSTOSE}

A CS pode ser causada por mutações $\begin{array}{lrr}\text { genéticas }^{25-27,28}, & \text { exposição } & \text { a } \\ \text { teratógenos } & & \\ & & \end{array}$ durante o período perinatal ${ }^{31,32}$, idade paterna e materna avançada, níveis educacionais dos pais ${ }^{33,34,38}$, uso de antidepressivos no período gestacional $^{36}$, estimulação endócrina pelos hormônios da tireóide ${ }^{37,38}$, terapia com metotrexato ${ }^{39}$ certas condições metabólicas ${ }^{25,26,40,41}$, uso excessivo de antiácido para o tratamento de cólica ${ }^{42}$, ingestão de certos nutrientes $^{43}$ e $\mathrm{o}$ uso de citrato de clomifeno ${ }^{44}$, portanto, o conhecimento da fisiopatologia que cada fator de risco possui é essencial para o entendimento da doença, sendo de necessidade para qualquer profissional da saúde que visa o tratamento ou prevenção da CS.

\section{Genética}

A mutação do gene que causa CS do tipo não sindrômica ocorre esporadicamente, mas assim como as do tipo sindrômicas, as alterações ocorrerem predominantemente em genes que sintetizam receptores de crescimento de fibroblastos $(F G F R)^{45}$, mas, existe também outros genes relacionados à CS não sindrômica, como TWIST $1^{27,28}$, FREM $1^{46}$, BBS9 ${ }^{47}$, LRIT3, EFNA4, RUNX2 ${ }^{48}$, ALX4 e IGFR1 ${ }^{49}$.

Quatro genes que codificam FGFR foram identificados, sendo que mutações em três destes genes, ou seja, FGFR1, FGFR2 e FGFR3, têm sido relacionadas principalmente nas formas mais comuns de CS sindrômica: Síndrome de Apert, Crouzon, Pfeiffer, Muenke ${ }^{50}$, além de ser relatado na literatura o aparecimento da síndrome de Prader-Willi com cariótipo de Klinefelter (XXY) e CS concomitantemente ${ }^{51}$. Nas CS ocasionadas pela síndrome de SaethreChotzen, as mutações ocorrem em um 
modulador dos FGFRs, denominado TWIST1 ${ }^{52}$; nas CS do tipo Boston o erro ocorre no MSX2(53) e na síndrome craniofrontonasal o defeito ocorre no gene EFNB1 $1^{54}$, mas, outros genes também estão ligados à CS do tipo sindrômica, como POR, RAB23, EFNA4 ${ }^{48,55}$, FAM20C, LMX1B ${ }^{56,57}, \mathrm{ESCO}^{5}$, GLI3, JAG1, KRAS, RECQL4, TGFBR1 ou TGFBR2 ${ }^{58}$.

A literatura descreve a respeito do fator de crescimento transformacional beta (TGF- $\beta$ ), sendo estes divididos em TGF- $\beta 1$, TGF- $\beta 2$ e TGF$\beta 3$, produzido estes pela dura-máter do animal, cuja função é exercer efeitos regulatórios na proliferação osteogênica nas suturas através de sua ligação à receptores transmembrânico da superfície celular de passagem única (FGFRs) ${ }^{59}$. Uma teoria foi proposta para explicar o modelo regulatório que tais fatores exercem sobre as suturas cranianas, na qual TGF- $\beta 1$ e TGF- $\beta 3$ produzem fatores que atuam como sinais indutivos ou de estabilização de outros sinais produzidos também pela dura-máter, que atuam no crescimento da calota craniana. Já TGF- $\beta 2$ atua na segregação dos sinais osteogênicos que promovem o recrutamento de osteoblastos e osteogênese frente ao osso, sendo essencial para o crescimento coordenado do cérebro e da calota craniana ${ }^{23,60,61}$. Outro modelo foi proposto para explicar a regulamentação da fusão das suturas (Figura 5), na qual o TGF- $\beta 2$ induz o fechamento precoce das suturas através da fosforilação da ERK 1 / 2 e esta, por sua vez, atua direta ou indiretamente na inibição da expressão da proteína Smad 2 / 3, que está associada com a permeabilidade da sutura. Como o ERK 1 / 2 é também um substrato para a sinalização do receptor FGFR, a regulação positiva da ERK 1 / 2 pode resultar também em uma facilitação para a fusão das suturas cranianas ${ }^{62,63}$.

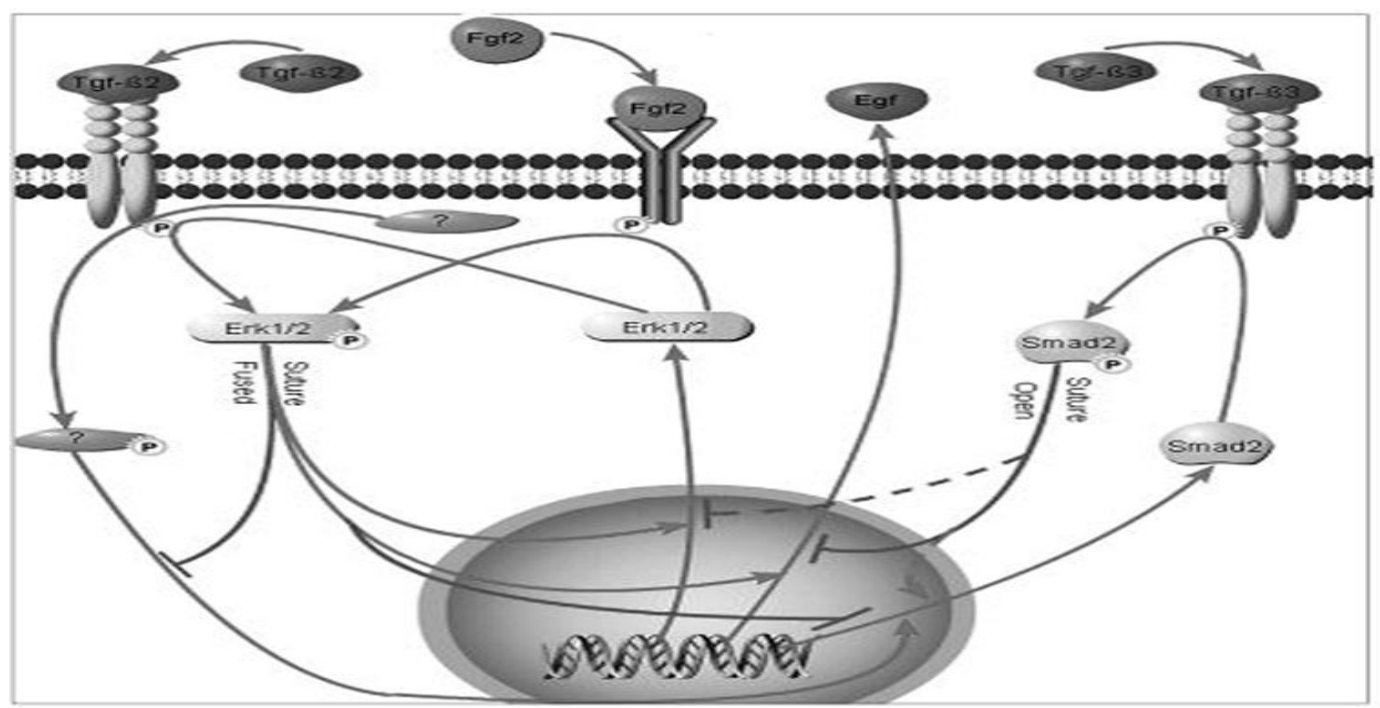

Figura 5. Modelo de sinalização de TGF-B2 em suturas. TGF-B2 regula a permeabilidade da fusão das suturas através da fosforilação de Erk $1 / 2$ e proteínas Smad2. As linhas contínuas indicam comprovados eventos de sinalização e a linha tracejada indica previu eventos de sinalização com base neste modelo. Modificada de Senarath-yapa et al. ${ }^{4}$

\section{Teratógenos}

O ácido retinóico (RA), um derivado da vitamina A que funciona como metabólito ativo na sinalização celular, tem capacidade de indução na diferenciação de diversas células ${ }^{64}$, sendo assim, tanto a deficiência quanto o excesso de RA têm amplos efeitos sobre a organogênese e embriogênse dos vertebrados ${ }^{65}$. Em particular, as anormalidades morfogenéticas craniofacial e de membros podem ser resultados da exposição ao
RA em períodos críticos no desenvolvimento esquelético fetal ${ }^{25,66,67}$.

Esta afirmação é comprovada ao analisar mutações na proteína Cyp26, pertencente à família do citocromo P450 oxidase e que tem a função de manter os níveis de RA basais através da transformação do mesmo em metabólitos biologicamente inativos ${ }^{68,69}$. Nesses casos, malformações estruturais tais como ossos longos que se ossificam através de um molde cartilaginoso (ossificação endocondral) bem 
como aqueles que se formam através de um tecido conjuntivo (ossificação membranosa) foram observados, associando-as ao alto nível de RA existente no organismo, uma vez que o mesmo não foi degradado pelo fígado ${ }^{70}$.

O mecanismo de ação do RA que causa CS ainda não está bem esclarecido, mas acredita-se que o RA ao se ligar nos receptores específicos para ácido retinóico (RAR) tem a capacidade de regular o desenvolvimento de vários genes importantes, incluindo os genes homeobox ${ }^{71-73}$. Nesses agrupamentos de genes, bem como outros, heterodímeros de RARY e do receptor retinóico X- $\alpha$ (RXR- $\alpha)$ reconhece e liga-se a elementos de DNA específicos (Rares) e induz a alterações epigenéticas, como a indução da transcrição de RA, alterando seus níveis ${ }^{74}$. Alterações nos níveis de RA pode estar associado também ao aumento dos níveis de proteínas coativadores como KAT3A (p300), KAT13B (pCIP), e da RNA polimerase II no rares $^{72,75}$.

O ácido valpróico, que foi usado para o tratamento de convulsões em mais de 30 anos, tem sido reconhecido como um teratógeno. Este fármaco aumenta a chance de má formação craniofaciais, entre elas a CS, de duas a sete vezes em fetos cuja mãe foi exposta à medicação, mas seu mecanismo de ação ainda não é muito bem esclarecido ${ }^{30,76-81}$.

\section{Tabagismo na gravidez}

Uma revisão sistemática realizada por Hackshaw et al. ${ }^{32}$, reuniu amostra desde 1959 até 2010, e concluiu que o fumo durante a gravidez traz sérios riscos para a saúde do bebê. $O$ fato é explicado porque os mais de 7000 produtos químicos presentes no cigarro, por exemplo, tem a capacidade de atravessar a barreira placentária e atuar diretamente de forma prejudicial sobre o feto. Os problemas mais comuns apresentados pelos fetos eram defeitos cardiovasculares, musculoesqueléticos e CS, defeitos faciais (face, olhos e ouvidos), do sistema gastrointestinal, genitourinário, do sistema respiratório e de pele $^{82-85}$.

Os mecanismos que levam a estas más formações ainda não são precisamente compreendidos, mas acredita-se que a nicotina causa redução do fluxo sanguíneo placentário devido sua ação vasoconstritora e o monóxido de carbono acarreta em uma menor disponibilidade de oxigênio aos tecidos fetais por se ligar a hemoglobina. Outras substâncias atuariam também perturbando a neoformação vascular e a função endotelial no materno, bem como as circulações fetais $^{83}$.

\section{Antidepressivos}

A associação entre o uso materno de antidepressivos, especialmente os inibidores seletivos da receptação de serotonina (SSRIs), durante a gravidez e defeitos congênitos em recém-nascidos tem sido um tema de muita discussão nos últimos $\operatorname{anos}^{86}$, isto porque a exposição aos SSRIs afeta a regulação do ciclo celular por aumentar o potencial proliferativo, além de diminuir a atividade apoptótica, levando a uma hiperplasia osteoblástica nas suturas, ocasionando o fechamento prematuro da mesma ${ }^{87}$. Os SSRIs analisados foram o citalopram, escitalopram, fluoxetina, paroxetina e sertralina, possuindo o primeiro SSRIs relação com defeitos de tubo neural; já com segundo, não foi encontrada nenhuma relação com defeitos nos recém-nascidos; entretanto, com o terceiro, foi relacionado defeitos de septo ventricular, do ventrículo direito, obstrução das vias de saída cardíaca e CS; com o quarto, anencefalia, defeitos de septos, do ventrículo direito, obstrução das vias de saída cardíaca, omphalocele e atresia de esôfago e com o quinto não foi encontrada nenhuma associação significante com defeitos de recém-nascidos ${ }^{36,88-90}$.

\section{Metotrexato}

O metotrexato é um antagonista do ácido fólico que inibe a dihidrofolato redutase, bloqueando, assim, a síntese de timidina e inibindo a síntese de DNA. Esta droga é muito utilizada na quimioterapia para o tratamento de câncer, mas também para tratar doenças reumáticas, dermatológicas, autoimunes, e doenças inflamatórias, além de sua utilização bem sucedida na gravidez ectópica ${ }^{91}$.

A exposição ao metotrexato durante o período pré-gestacional está relacionado a másformações congênitas como defeitos septais atriais, tetralogia de Fallot, estenose pulmonar valvar, defeitos do septo ventricular, fenda palatina, hipospadia, hérnia diafragmática congênita, microtia e CS, entretanto, o mecanismo fisiopatológico relacionado a CS não é muito bem esclarecido ${ }^{92-94}$.

Idade avançada e níveis de instrução dos pais 
A idade avançada dos pais e o risco de desenvolvimento de CS no feto ainda não são claros, mas a explicação se baseia na associação da idade com o aumento das mutações no DNA e aberrações cromossômicas nos espermatozóides $^{95}$. Devido o aumento das mutações no DNA dos espermatozoides, homens mais velhos estão em maior risco de gerar crianças com CS coronal, mas não sagital ou metópica ${ }^{1,31,96}$.

A literatura se contradiz a respeito dos níveis de instrução dos pais como um fator de risco para o desenvolvimento de CS, existindo estudos que encontraram associação entre ambos ${ }^{37,43,97}$ e outros que não encontraram nenhuma associação ${ }^{7,31,98}$. Mas suponha-se que o maior nível de instrução diminuiria a incidência da má formação, uma vez que um maior conhecimento sobre os fatores causais levaria a uma maior autonomia dos indivíduos em prevenir sua exposição aos fatores que podem acarretar na doença, entretanto, maiores pesquisas são necessárias ${ }^{96}$.

\section{Hormônios Tireoidianos}

Durante 0 desenvolvimento fetal, o hormônio liberador de tirotropina (TRH) e o hormônio estimulante da tireóide (TSH) são secretados entre a $18^{\mathrm{a}}$ e 20 a semana de gestação. Esses hormônios são produzidos na hipófise anterior e fazem com que as células foliculares captem tireoglobulina iodada do lúmem de cada folículo tireoidiano por endocitose e, em seguida, a submetem à digestão lisossomal, formando como produto T3 (triiodotironina) e T4( tiroxina), que tem inúmeras funções, entre elas, estimular o crescimento celular ${ }^{99,100}$.

Por possuir a capacidade de atravessar a barreira placentária, os hormônios tireoidianos são capazes de alterar vários genes no feto, estando entre eles genes que controlam a osteogênese da calota craniana, como o FGF, IGF e TGF- $\beta$, possuindo tais alterações a capacidade de levar a uma hiperexpressão do gene, causando uma proliferação celular nas suturas cranianas, levando à $\mathrm{CS}^{38,101,102}$.

\section{Raquitismo Hipofosfatêmico}

A CS pode estar relacionada com um distúrbio metabólico, como a deficiência do fosfato ${ }^{103,104}$, sendo o raquitismo ligado ao $X$ hipofosfatêmico (XLH), conhecido como raquitismo resistente de vitamina $\mathrm{D}$ (VDDR), a causa metabólica mais comum da fusão prematura das suturas cranianas ${ }^{105}$.

A patogênese da CS no raquitismo hipofosfatêmico foi estudada em ratos que possuíam mutações ligadas ao cromossomo X. Ela ilustra a diferença entre as mutações no gene PHEX contra as mutações do receptor do fator de crescimento de fibroblastos, observados em outras formas de $\mathrm{CS}^{41,106}$. O gene PHEX defeituoso pode desencadear distúrbios na mineralização óssea através da interação com FGF-2 e 3, além do gene EFEM; isso conduz à desregulação do equilíbrio do fosfato e causa a inibição da mineralização óssea, respectivamente, tornando a criança altamente suscetível à $\mathrm{CS}^{107}$.

Portanto, mutações na proteína Phex que regula 0 fosfato causa raquitismo hipofosfatêmico ligado ao X, e esta comorbidade está relacionada à CS pela defeituosa mineralização óssea que ocorre como consequência dos baixos níveis séricos de fosfato $^{108}$. A literatura mostra também que mutações que inativam a enzima fosfatase alcalina não específica (TNAP), geradora enzimática do fosfato inorgânico e o essencial promotor da mineralização nos tecidos, levam à CS, mas os mecanismos que produzem a CS por diminuição da ação enzimática ainda não é conhecido ${ }^{108}$.

\section{Nutrientes}

O estado nutricional de uma mulher grávida tem sido identificado como etilogia de diversas malformações estruturais, entre elas a $\mathrm{CS}^{109}$. Acredita-se que nutrientes associados com o metabolismo de um carbono, como riboflavina e vitamina B6, e de dois nutrientes antioxidantes, vitamina $E$ e $C$, em excesso pode estar relacionado com o aparecimento da moléstia apresentada $^{43}$.

A literatura diverge-se com respeito ao uso de ácido fólico no primeiro trimestre de gravidez, Selber et al, 2008, afirma que o uso de ácido fólico pode estar associado com o aumento do risco para CS, uma vez que, as regiões estudadas com os maiores índices de CS foram onde as mulheres mais utilizavam o ácido fólico ${ }^{110}$. Entretanto, Carmichael et al. ${ }^{43}$, não encontrou associação entre suplementos que continham ácido fólico, bem como o mesmo utilizado de forma independente, e o aumento de risco para CS. Com isto, maiores pesquisas a respeito de CS associado com ácido fólico são necessárias. 


\section{Uso de Citrato de clomifeno}

O citrato de clomifeno é uma droga não esteróide indutor da ovulação que é usado em seres humanos a mais de 40 anos $^{111}$. Entretanto, a literatura mostra que o uso desse fármaco esta relacionado a diversas más formações, entre elas a CS, sendo que o mecanismo de ação da droga que leva ao aumento do risco ainda é desconhecido ${ }^{44}$.

Mesmo com os diversos fatores causais associados à CS encontrados, diversas outras pesquisas acabaram não sendo incluída, isto por motivos de limitações, entre elas, podemos citar: estudos cujas conclusões foram inconsistentes e a falta de artigos que explicassem os mecanismos de como os fatores de risco causa a CS.

\section{CONCLUSÃO}

O presente estudo conclui que 0 conhecimento dos fatores causais é fundamental para a definição de estratégias que visem à diminuição da incidência através da autonomia do individuo, abordando como possíveis estratégias 0 aconselhamento genético, a diminuição da exposição aos teratógenos, alimentação diferenciada, bem como elucidar aos consumidores que certos tipos de produtos podem acarretar em más formações congênitas. Com isto, apresenta-se claro que somente após o conhecimento dos fatores causais e seu método de ação é possuímos um ponto de partida da qual possamos traçar estratégias futuras que visam uma diminuição da CS.

\section{CONFLITOS DE INTERESSE}

Os autores declaram não haver qualquer potencial conflito de interesse que possa interferir na imparcialidade deste trabalho científico.

\section{REFERÊNCIAS}

1. Lajeunie E, Le Merrer M, Bonaïti-Pellie C, Marchac D, Renier D. Genetic study of nonsyndromic craniosynostosis. Am J Med Genet Banner. 1995;55(4):500-4. DOI: https://doi.org/10.1002/ajmg.1320550422

2. Gracia A, Arsuaga JL, Martínez I, Lorenzo C, Carretero JM, Bermúdez de Castro JM, et al. Craniosynostosis in the Middle Pleistocene human Cranium 14 from the Sima de los Huesos, Atapuerca, Spain. Proc Natl Acad Sci U S A. 2009;106(16):6573-8. DOI: https://doi.org/10.1073/pnas.0900965106

Colloq Vitae 2018 mai-ago; 10(2): 65-77. DOI: 10.5747/cv.2018.v10.n2.v234 ISSN 1984-6436/C 2018 - Publicado pela Universidade do Oeste Paulista.

Artigo Open Access sob uma licença CC BY-NC-ND (http://creativecommons.org/licenses/by-nc-nd/4.0/).
3. Otto, Adolph Wilhelm. Lehrbuch der pathologischen Anatomie des Menschen und der Thiere. 1786-1845.

4. Senarath-yapa K, Chung MT, Mcardle A, Wong VW, Quarto N, Longaker MT et al. Molecular pathways and future pharmacologic therapy Craniosynostosis. Organogenesis. 2012;8:103-13.

https://doi.org/10.4161/org.23307

5. Crouzon O. La dysostose cranio-faciale héréditaire. Bull Mem Soc Anthropol Paris. 1935;6(4):41-5. DOI: https://doi.org/10.3406/bmsap.1935.9331

6. Tessier P. The treatment of facial dysmorphy peculiary to cranio-facial dysostosis (C.F.D.). Crouzon and Apert diseases. Total osteotomy and sagittal displacement of the facial mass. Chir mémoires l'Académie Chir. 1970;96(10):667-74. DOI: https://doi.org/10.1002/ajmg.a.32208

7. Boulet SL, Rasmussen SA, Honein MA. A populationbased study of craniosynostosis in metropolitan Atlanta, 1989-2003. Am J Med Genet A. 2008. 146a(8):984-81.

DOI: https://doi.org/10.1002/ajmg.a.32208

8. David L, Glazier S, Pyle J, Thompson J, Argenta L. Classification System for Sagittal Craniosynostosis. J Craniofacial Surg. 2009;20(2):279-82. DOI: https://doi.org/10.1097/SCS.0b013e3181945ab0

9. Rice DP. Craniofacial sutures: development, disease and treatment. 2008. 236p. DOI: https://doi.org/10.1159/isbn.978-3-8055-8152-3

10. Kimonis V, Gold J-A, Hoffman TL, Panchal J, Boyadjiev SA. Genetics of craniosynostosis. Semin Pediatr Neurol. 2007;14(3):150-61. DOI: https://doi.org/10.1016/j.spen.2007.08.008

11. Connolly JP, Gruss J, Seto, MLBS, Whelan MF, Ellenbogen R, Weiss A, Buchman SR, Cunningham ML. Progressive postnatal craniosynostosis and increased intracranial pressure. Plast Reconstr Surg. 2004;113(5):1313-23. DOI: https://doi.org/10.1097/01.PRS.0000111593.96440.30

12. Hoefkens MF, Vermeij-Keers C, Vaandrager JMM. Crouzon syndrome: phenotypic signs and symptoms of the postnatally expressed subtype. J Craniofac Surg. 2004;15(2):233-40.

DOI: https://doi.org/10.1097/00001665-200403000-00014

13. Cohen MM, Ruth ME. Craniosynostosis: diagnosis, evaluation, and management. $2^{\text {nd }}$ ed. Oxford University Press; 2000.2480 . DOI: https://doi.org/10.1002/(SICI)10968628(19960329)62:3<282::AID-AJMG15>3.0.CO;2-G 
14. Lajeunie E, Le Merrer M, Bonaïti-Pellie C, Marchac D RD. Genetic study of scaphocephaly. Am J Med Genet. 1996;62(3):282-5. DOI: https://doi.org/10.1002/(SICI)10968628(19960329)62:3<282::AID-AJMG15>3.0.CO;2-G

15. Slater BJ, Lenton KA, Kwan MD, Gupta DM, Wan DC, Longaker MT. Cranial sutures: a brief review. Plast Reconstr Surg. 2008;121(4):170e-8e. DOI: https://doi.org/10.1097/01.prs.0000304441.99483.97

16. Hunter AG, Rudd NL. Craniosynostosis. II. Coronal synostosis: its familial characteristics and associated clinical findings in 109 patients lacking bilateral polysyndactyly or syndactyly. Teratology. 1977;15(3):301-9. DOI: https://doi.org/10.1002/tera.1420150312

17. Lajeunie E, Le Merrer M, Marchac D RD. Syndromal and nonsyndromal primary trigonocephaly: analysis of a series of 237 patients. Am J Med Genet. 1998;75(2):211-5. DOI: https://doi.org/10.1002/(SICI)10968628(19980113)75:2<211::AID-AJMG19>3.0.CO;2-S

18. FS Jehee, D Johnson, LG Alonso, DP Cavalcanti, E De Sá Moreira, FL Alberto, F Kok, C Kim, SA Wall, EW Jabs, SA Boyadjiev AW and MP-B. Molecular screening for microdeletions at 9p22-p24 and 11q23-q24 in a large cohort of patients with trigonocephaly. J Genet Mol Pers Med. 2005;67(6):503-10. DOI: https://doi.org/10.1111/j.1399-0004.2005.00438.x

19. Huang MH, Gruss JS, Clarren SK, Mouradian WE, Cunningham ML, Roberts TS, et al. The differential diagnosis of posterior plagiocephaly: true lambdoid synostosis versus positional molding. Plast Reconstr Surg. 1996;98(5):765-74. DOI: https://doi.org/10.1097/00006534-199610000-00001

20. Wilkie AOM, Byren JC, Hurst JA, Jayamohan J, Johnson D, Knight SJL, et al. Prevalence and complications of single-gene and chromosomal disorders in craniosynostosis. Pediatrics. 2010;126(2):e391-400. DOI: https://doi.org/10.1542/peds.2009-3491

21. Rekate HL. Occipital plagiocephaly: a critical review of the literature. J Neurosurg. $1998 ; 89(1): 24-30$. DOI: https://doi.org/10.3171/ins.1998.89.1.0024

22. Fryburg JS, Hwang V, Lin KY. Recurrent lambdoid synostosis within two families. Am J Med Genet. 1995;58(3):262-6. DOI: https://doi.org/10.1002/ajmg.1320580313

23. Opperman LA. Cranial sutures as intramembranous bone growth sites. Dev Dyn. 2000;219(4):472-85. DOI: Colloq Vitae 2018 mai-ago; 10(2): 65-77. DOI: 10.5747/cv.2018.v10.n2.v234 ISSN 1984-6436/@ 2018 - Publicado pela Universidade do Oeste Paulista.

Artigo Open Access sob uma licença CC BY-NC-ND (http://creativecommons.org/licenses/by-nc-nd/4.0/). https://doi.org/10.1002/1097-

0177(2000)9999:9999\%3C::AID-

DVDY1073\%3E3.0.CO;2-F

24. Coussens AK, Wilkinson CR, Hughes IP, Morris CP, van Daal A, Anderson PJ, et al. Unravelling the molecular control of calvarial suture fusion in children with craniosynostosis. BMC Genomics. 2007;8(1):458. DOI: https://doi.org/10.1186/1471-2164-8-458

25. Yip JE, Kokich VG, Shepard TH. The effect of high doses of retinoic acid on prenatal craniofacial development in Macaca nemestrina. Teratology. 1980;21(1):29-38. DOI: https://doi.org/10.1002/tera.1420210105

26. Cohen MM. Sutural biology and the correlates of craniosynostosis. Am J Med Genet. 1993;47(5):581616. DOI: https://doi.org/10.1002/ajmg.1320470507

27. Robin NH, Falk MJ, Haldeman-Englert CR. FGFRRelated Craniosynostosis Syndromes. University of Washington, Seattle; 2011.

28. Melville H, Wang Y, Taub PJ, Jabs EW. Genetic basis of potential therapeutic strategies for craniosynostosis. Am J Med Genet A. 2010; 152A(12):3007-15. DOI: https://doi.org/10.1002/ajmg.a.33703

29. Winter RM, Donnai D, Burn J, Tucker SM. Fetal valproate syndrome: is there a recognisable phenotype? J Med Genet. 1987;24(11):692-5. DOI: https://doi.org/10.1136/jmg.24.11.692

30. Jentink J, Loane MA, Dolk H, Barisic I, Garne E, Morris JK, et al. Valproic acid monotherapy in pregnancy and major congenital malformations. N Engl J Med. 2010;362(23):2185-93. DOI: https://doi.org/10.1056/NEJMoa0907328

31. Källén K. Maternal smoking and craniosynostosis. Teratology. 1999;60(3):146-50. DOI: https://doi.org/10.1002/(SICI)10969926(199909)60:3<146::AID-TERA10>3.0.CO;2-3

32. Hackshaw A, Rodeck C, Boniface S. Maternal smoking in pregnancy and birth defects: A systematic review based on 173687 malformed cases and 11.7 million controls. Hum Reprod Update. 2011;17(5):589604. DOI: https://doi.org/10.1093/humupd/dmr022

33. Bradley CM, Alderman BW, Williams MA, Checkoway H, Fernbach SK, Greene C, Bigelow PL RJ. Parental occupations as risk factors for craniosynostosis in offspring. Epidemiology. 1995;6(3):306-10. DOI: https://doi.org/10.1097/00001648-199505000-00020 
34. Alderman BW, Lammer EJ, Joshua SC, Cordero JF, Ouimette DR, Wilson MJ, et al. An epidemiologic study of craniosynostosis: risk indicators for the occurrence of craniosynostosis in Colorado. Am J Epidemiol. 1988;128(2):431-8. DOI: https://doi.org/10.1093/oxfordjournals.aje.a114983

35. Källén BR-GE. Maternal drug use, fertility problems, and infant craniostenosis. Cleft Palate Craniofacial J. 2005;42(6):589-93. DOI: https://doi.org/10.1597/04-031.1

36. Reefhuis J, Devine O, Friedman JM, Louik C, Honein MA, for the National Birth Defects Prevention Study. Specific SSRIs and birth defects: bayesian analysis to interpret new data in the context of previous reports. $\mathrm{Br}$ Med J. 2015;350:h3190. DOI: https://doi.org/10.1136/bmj.h3190

37. Rasmussen SA, Yazdy MM, Carmichael SL, Jamieson DJ, Canfield MA, Honein MA. Maternal thyroid disease as a risk factor for craniosynostosis. Obstet Gynecol. 2007;110(2Pt1):369-77. DOI: https://doi.org/10.1097/01.AOG.0000270157.88896.7 6

38. Cray JJ, Khaksarfard K, Weinberg SM, Elsalanty M, Yu JC. Effects of Thyroxine Exposure on Osteogenesis in Mouse Calvarial Pre-Osteoblasts. PLoS One. 2013;8(7):1-9.

DOI: https://doi.org/10.1371/journal.pone.0069067

39. Dawson AL, Riehle-Colarusso T, Reefhuis J, Arena JF. Maternal exposure to methotrexate and birth defects: a population-based study. Am J Med Genet A. 2014;164(9):2212-16.

DOI:

https://doi.org/10.1002/ajmg.a.36625

40. Reilly BJ, Leeming JM. Craniosynostosis in the rachitic spectrum. J Pediatr. 1964;64:396-405. DOI: https://doi.org/10.1053/spen.2002.32504

41. Roy WA, lorio RJ, Meyer GA. Craniosynostosis in vitamin D-resistant rickets. A mouse model. J Neurosurg. 1981;55(2):265-71. DOI: https://doi.org/10.3171/ins.1981.55.2.0265

42. Pivnick EK, Kerr NC, Kaufman RA, Jones DP CR. Rickets secondary to phosphate depletion. A sequela of antacid use in infancy. Clin Pediatr (Phila). 1995;34(2):73-8.

DOI: https://doi.org/10.1177/000992289503400202

43. Carmichael SL, Rasmussen SA, Lammer EJ, Ma C, Shaw GM. Craniosynostosis and nutrient intake during pregnancy. Birth Defects Res Part A - Clin Mol Teratol. 2010;88(12):1032-9.

DOI:

https://doi.org/10.1002/bdra.20717

Colloq Vitae 2018 mai-ago; 10(2): 65-77. DOI: 10.5747/cv.2018.v10.n2.v234 ISSN 1984-6436/@ 2018 - Publicado pela Universidade do Oeste Paulista.

Artigo Open Access sob uma licença CC BY-NC-ND (http://creativecommons.org/licenses/by-nc-nd/4.0/).
44. Reefhuis J, Honein MA, Schieve LA, Rasmussen SA. Use of clomiphene citrate and birth defects, National Birth Defects Prevention Study, 19972005. Hum Reprod. 2011;26(2):451-7. DOI: https://doi.org/10.1093/humrep/deq313

45. Glaser RL, Jiang W, Boyadjiev SA, Tran AK, Zachary $A A$, Van Maldergem L, et al. Paternal origin of FGFR2 mutations in sporadic cases of Crouzon syndrome and Pfeiffer syndrome. Am J Hum Genet. 2000;66(3):76877. DOI: https://doi.org/10.1086/302831

46. Vissers LELM, Cox TC, Maga AM, Short KM, Wiradjaja F, Janssen IM, et al. Heterozygous mutations of FREM1 are associated with an increased risk of isolated metopic craniosynostosis in humans and mice. PLoS Genet. 2011;7(9):e1002278. DOI: https://doi.org/10.1371/journal.pgen.1002278

47. Justice CM, Yagnik G, Kim Y, Peter I, Jabs EW, Erazo $M$, et al. A genome-wide association study identifies susceptibility loci for nonsyndromic sagittal craniosynostosis near BMP2 and within BBS9. Nat Genet. 2012;44(12):1360-4. DOI: https://doi.org/10.1038/ng.2463

48. Pandey RK, Ali A, Singh A, Gayan S BM. Methylenetetrahydrofolate reductase $\mathrm{C677T}$ variant in Indian children with craniosynostosis: Its role in the pathogenesis, risk of craniosynostosis. Indian J Hum Genet. 2014;20(2):155-9. DOI: https://doi.org/10.2298/GENSR1303881P

49. Heuzé Y, Holmes G, Peter I, Richtsmeier JT, Jabs EW. Closing the Gap: Genetic and Genomic Continuum from Syndromic to Nonsyndromic Craniosynostoses. Curr Genet Med Rep. 2014;2(3):135-45. DOI: https://doi.org/10.1007/s40142-014-0042-x

50. Guagnano V, Kauffmann A, Wöhrle S, Stamm C, Ito $M$, Barys $L$, et al. FGFR genetic alterations predict for sensitivity to NVP-BGJ398, a selective pan-FGFR inhibitor. Cancer Discov. 2012;2(12):1118-33. DOI: https://doi.org/10.1158/2159-8290.CD-12-0210

51. Carvalho DR, Trad CS, Pina-Neto JM. Atypical presentation of Prader-Willi syndrome with Klinefelter (XXY karytype) and craniosynostosis. Arq Neuropsiquiatr. 2006;64(2A):303-5. DOI: http://dx.doi.org/S0004-282X2006000200024

52. Howard TD, Paznekas WA, Green ED, Chiang LC, Ma N, Ortiz de Luna RI, Garcia Delgado C, GonzalezRamos M, Kline AD JE. Mutations in TWIST, a basic helix-loop-helix transcription factor, in SaethreChotzen syndrome. Nat Genet. 1997;15(1):36-41. DOI: https://doi.org/10.1038/ng0197-36

53. Janssen A, Hosen MJ, Jeannin P, Coucke PJ, De 
Paepe A, Vanakker OM. Second family with the boston-type craniosynostosis syndrome: Novel mutation and expansion of the clinical spectrum. Am J Med Genet Part A. 2013;161(9):2352-7. DOI: https://doi.org/10.1002/ajmg.a.36077

54. Wieland I, Makarov R, Reardon W, Tinschert S, Goldenberg A, Thierry $P$, et al. Dissecting the molecular mechanisms in craniofrontonasal syndrome: differential mRNA expression of mutant EFNB1 and the cellular mosaic. Eur J Hum Genet. 2008;16(2):18491. DOI: https://doi.org/10.1038/sj.ejhg.5201968

55. Passos-Bueno MR, Serti Eacute AEAL, Jehee FS, Fanganiello R, Yeh E. Genetics of craniosynostosis: genes, syndromes, mutations and genotypephenotype correlations. Front Oral Biol. 2008;12:10743. DOI: https://doi.org/10.1159/000115035

56. Sweeney E, Fryer A, Mountford R, Green A, Mclntosh I. Nail patella syndrome: a review of the phenotype aided by developmental biology. J Med Genet. 2003;40(3):153-62. DOI: https://doi.org/10.1136/jmg.40.3.153

57. Simpson MA, Scheuerle A, Hurst J, Patton MA, Stewart $\mathrm{H}$, Crosby AH. Mutations in FAM20C also identified in non-lethal osteosclerotic bone dysplasia. Clin Genet. 2009;75(3):271-6. DOI: https://doi.org/10.1111/j.1399-0004.2008.01118.x

58. Johnson D, Wilkie AOM. Craniosynostosis. Eur J Hum Genet. 2011;19(4):369-76. DOI: https://doi.org/10.1038/ejhg.2010.235

59. Opperman LA, Passarelli RW, Morgan EP, Reintjes $M$, Ogle RC. Cranial sutures require tissue interactions with dura mater to resist osseous obliteration in vitro. J Bone Miner Res. 1995;10(12):1978-87. DOI: https://doi.org/10.1002/jbmr.5650101218

60. Gosain AK, Machol JA, Gliniak C, Halligan NLN. TGFbeta1 RNA interference in mouse primary dura cell culture: downstream effects on TGF receptors, FGF-2, and FGF-R1 mRNA levels. Plast Reconstr Surg. 2009;124(5):1466-73. DOI: https://doi.org/10.1097/PRS.0b013e3181b98947

61. Gupta AK, Eshraghi Y, Gliniak C, Gosain AK. Nonviral transfection of mouse calvarial organ in vitro using Accell-modified siRNA. Plast Reconstr Surg. 2010;125(2):494-501. DOI: https://doi.org/10.1097/PRS.0b013e3181c82df1

62. Opperman LA, Fernandez CR, So S, Rawlins JT. Erk1/2 signaling is required for Tgf-beta 2-induced suture closure. Dev Dyn. 2006;235(5):1292-9. DOI: https://doi.org/10.1002/dvdy.20656

Colloq Vitae 2018 mai-ago; 10(2): 65-77. DOI: 10.5747/cv.2018.v10.n2.v234 ISSN 1984-6436/C 2018 - Publicado pela Universidade do Oeste Paulista.

Artigo Open Access sob uma licença CC BY-NC-ND (http://creativecommons.org/licenses/by-nc-nd/4.0/).
63. Lee S-W, Choi K-Y, Cho J-Y, Jung S-H, Song K-B, Park $\mathrm{E}-\mathrm{K}$, et al. TGF-beta2 stimulates cranial suture closure through activation of the Erk-MAPK pathway. J Cell Biochem. 2006;98(4):981-91. DOI: https://doi.org/10.1002/icb.20773

64. Urvalek A, Laursen KB, Gudas LJ. The roles of retinoic acid and retinoic acid receptors in inducing epigenetic changes. Subcell Biochem. 2014;70:129-49. DOI: https://doi.org/10.1007/978-94-017-9050-5 7

65. Duester G. Retinoic acid synthesis and signaling during early organogenesis. Cell. 2008;134(6):921-31. DOI: https://doi.org/10.1016/j.cell.2008.09.002

66. Kwasigroch TE, Bullen M. Effects of isotretinoin (13-cis-retinoic acid) on the development of mouse limbs in vivo and in vitro. Teratology. 1991;44(6):60516. DOI: https://doi.org/10.1002/tera.1420440603

67. James AW, Levi $B, X u Y$, Carre AL, Longaker MT. Retinoic acid enhances osteogenesis in cranial suturederived mesenchymal cells: potential mechanisms of retinoid-induced craniosynostosis. Plast Reconstr Surg. 2010;125(5):1352-61. DOI: https://doi.org/10.1097/PRS.0b013e3181d62980

68. Fujii H, Sato T, Kaneko S, Gotoh O, Fujii-Kuriyama $\mathrm{Y}$, Osawa K, et al. Metabolic inactivation of retinoic acid by a novel P450 differentially expressed in developing mouse embryos. EMBO J. 1997;16(14):4163-73. https://doi.org/10.1093/emboj/16.14.4163

69. White JA, Beckett-Jones B, Guo YD, Dilworth FJ, Bonasoro J, Jones $\mathrm{G}$, et al. cDNA cloning of human retinoic acid-metabolizing enzyme (hP450RAl) identifies a novel family of cytochromes P450. J Biol Chem. 1997;272(30):18538-41. DOI: https://doi.org/10.1074/jbc.272.30.18538

70. Laue K, Pogoda HM, Daniel PB, Van Haeringen A, Alanay $Y$, Von Ameln $S$, et al. Craniosynostosis and multiple skeletal anomalies in humans and zebrafish result from a defect in the localized degradation of retinoic acid. Am J Hum Genet. 2011;89(5):595-606. DOI: https://doi.org/10.1016/j.ajhg.2011.09.015

71. Langston AW, Thompson JR, Gudas LJ. Retinoic acid-responsive enhancers located $3^{\prime}$ of the Hox $A$ and Hox B homeobox gene clusters. Functional analysis. J Biol Chem. 1997;272(4):2167-75. DOI: https://doi.org/10.1074/jbc.272.4.2167

72. Kashyap V, Gudas LJ, Brenet F, Funk P, Viale A, Scandura JM. Epigenomic reorganization of the clustered Hox genes in embryonic stem cells induced by retinoic acid. J Biol Chem. 2011;286(5):3250-60. DOI: https://doi.org/10.1074/jbc.M110.157545 
73. Kashyap V, Laursen KB, Brenet $F$, Viale AJ, Scandura JM, Gudas LJ. RAR is essential for retinoic acid induced chromatin remodeling and transcriptional activation in embryonic stem cells. J Cell Sci. 2012;126(4):999-1008. DOI: https://doi.org/10.1242/ics.119701

74. Gillespie RF, Gudas LJ. Retinoid regulated association of transcriptional co-regulators and the polycomb group protein SUZ12 with the retinoic acid response elements of Hoxa1, RARbeta(2), and Cyp26A1 in F9 embryonal carcinoma cells. J Mol Biol. 2007;372(2):298-316.

DOI: https://doi.org/10.1016/i.jmb.2007.06.079

75. Laursen KB, Mongan NP, Zhuang Y, Ng MM, Benoit YD, Gudas LJ. Polycomb recruitment attenuates retinoic acid-induced transcription of the bivalent NR2F1 gene. Nucleic Acids Res. 2013 J;41(13):6430-43. DOI: https://doi.org/10.1093/nar/gkt367

76. Pinder RM, Brogden RN, Speight TM, Avery GS. Sodium valproate: a review of its pharmacological properties and therapeutic efficacy in epilepsy. Drugs. 1977;13(2):81-123.

DOI: https://doi.org/10.2165/00003495-197713020-00001

77. Hiilesmaa VK, Bardy AH, Granström ML, Teramo KA. Valproic acid during pregnancy. Lancet (London, England). 1980;1(8173):883. DOI: https://doi.org/10.1016/S0140-6736(80)91387-2

78. Gomez MR. Possible teratogenicity of valproic acid. J Pediatr. 1981;98(3):508-9. DOI: https://doi.org/10.1016/S0022-3476(81)80743-3

79. Robert E, Guibaud P. Maternal valproic acid and congenital neural tube defects. Lancet (London, England). 1982;2(8304):937. DOI: https://doi.org/10.1016/S0140-6736(82)90908-4

80. Löscher W, Nau H, Marescaux C, Vergnes M. Comparative evaluation of anticonvulsant and toxic potencies of valproic acid and 2-en-valproic acid in different animal models of epilepsy. Eur J Pharmacol. 1984;99(2-3):211-8.

DOI:

https://doi.org/10.1016/0014-2999(84)90243-7

81. Lindhout D, Omtzigt JG, Cornel MC. Spectrum of neural-tube defects in 34 infants prenatally exposed to antiepileptic drugs. Neurology. 1992;42(4 Suppl 5):111-8.

82. Werler MM, Pober BR, Holmes LB. Smoking and pregnancy. Teratology. 1985;32(3):473-81. DOI: https://doi.org/10.1002/tera.1420320316

83. Quinton $A E$, Cook C-M, Peek MJ. The relationship Colloq Vitae 2018 mai-ago; 10(2): 65-77. DOI: 10.5747/cv.2018.v10.n2.v234 ISSN 1984-6436/@ 2018 - Publicado pela Universidade do Oeste Paulista.

Artigo Open Access sob uma licença CC BY-NC-ND (http://creativecommons.org/licenses/by-nc-nd/4.0/). between cigarette smoking, endothelial function and intrauterine growth restriction in human pregnancy. BJOG. 2008;115(6):780-4. DOI: https://doi.org/10.1111/i.1471-0528.2008.01691.x

84. Talbot P. In vitro assessment of reproductive toxicity of tobacco smoke and its constituents. Birth Defects Res C Embryo Today. 2008;84(1):61-72. DOI: https://doi.org/10.1002/bdrc.20120

85. Rogers JM. Tobacco and pregnancy. Reprod Toxicol. 2009;28(2):152-60. DOI: https://doi.org/10.1016/i.reprotox.2009.03.012

86. FDA - Advising of Risk of Birth Defects with Paxil. 2005

87. Cray JJ, Weinberg SM, Parsons TE, Howie RN, Elsalanty M, Yu JC. Selective serotonin reuptake inhibitor exposure alters osteoblast gene expression and craniofacial development in mice. Birth Defects Res A Clin Mol Teratol. 2014;100(12):912-23. DOI: https://doi.org/10.1002/bdra.23323

88. Alwan S, Reefhuis J, Rasmussen SA, Olney RS, Friedman JM. Use of selective serotonin-reuptake inhibitors in pregnancy and the risk of birth defects. N Engl J Med. 2007;356(26):2684-92. DOI: https://doi.org/10.1056/NEJMoa066584

89. Louik C, Lin AE, Werler MM, Hernández-Díaz S, Mitchell AA. First-trimester use of selective serotoninreuptake inhibitors and the risk of birth defects. N Engl J Med. 2007;356(26):2675-83. DOI: https://doi.org/10.1056/NEJMoa067407

90. Malm H, Artama M, Gissler M, Ritvanen A. Selective serotonin reuptake inhibitors and risk for major congenital anomalies. Obstet Gynecol. 2011;118(1):111-20. DOI: https://doi.org/10.1097/AOG.0b013e318220edcc

91. Lloyd ME, Carr M, McElhatton P, Hall GM, Hughes RA. The effects of methotrexate on pregnancy, fertility and lactation. QJM. 1999;92(10):551-63. DOI: https://doi.org/10.1093/qjmed/92.10.551

92. Piggott KD, Sorbello A, Riddle E, DeCampli W. Congenital cardiac defects: a possible association of aminopterin syndrome and in utero methotrexate exposure? Pediatr Cardiol. 2011;32(4):518-20. DOI: https://doi.org/10.1007/s00246-011-9913-z

93. Poggi SH, Ghidini A. Importance of timing of gestational exposure to methotrexate for its teratogenic effects when used in setting of misdiagnosis of ectopic pregnancy. Fertil Steril. 2011;96(3):669-71. DOI: 
94. Hyoun SC, Običan SG, Scialli AR. Teratogen update: methotrexate. Birth Defects Res A Clin Mol Teratol. 2012;94(4):187-207. DOI: https://doi.org/10.1002/bdra.23003

95. Green RF, Devine O, Crider KS, Olney RS, Archer N, Olshan AF, et al. Association of paternal age and risk for major congenital anomalies from the National Birth Defects Prevention Study, 1997 to 2004. Ann Epidemiol. 2010;20(3):241-9. DOI: https://doi.org/10.1016/j.annepidem.2009.10.009

96. Barik M, Bajpai M, Das RR, Panda SS. Study of environmental and genetic factors in children with craniosynostosis: a case-control study. J Pediatr Neurosci. 2013;8(2):89-92. DOI: https://doi.org/10.4103/1817-1745.117833

97. Zeiger JS, Beaty TH, Hetmanski JB, Wang H, Scott $A F$, Kasch L, et al. Genetic and environmental risk factors for sagittal craniosynostosis. J Craniofac Surg. 2002;13(5):602-6.

DOI: http://dx.doi.org/10.1097/01.SCS.0000026407.16202. OE

98. Reefhuis J, Honein MA, Shaw GM, Romitti PA. Fertility treatments and craniosynostosis: California, Georgia, and lowa, 1993-1997. Pediatrics. 2003;111(5 Pt 2):1163-6. DOI: http://dx.doi.org/10.1542/peds.111.5.S1.1163

99. Brent GA. Tissue-specific actions of thyroid hormone: insights from animal models. Rev Endocr Metab Disord. 2000;1(1-2):27-33. DOI: https://doi.org/10.1023/A:1010056202122

100. Bassett JHD, Williams AJ, Murphy E, Boyde A, Howell PGT, Swinhoe R, et al. A lack of thyroid hormones rather than excess thyrotropin causes abnormal skeletal development in hypothyroidism. Mol Endocrinol. 2008;22(2):501-12. DOI: https://doi.org/10.1210/me.2007-0221

101. Akita S, Hirano A, Fujii T. Identification of IGF-I in the calvarial suture of young rats: histochemical analysis of the cranial sagittal sutures in a hyperthyroid rat model. Plast Reconstr Surg. 1996;97(1):1-12. DOI: https://doi.org/10.1097/00006534-199601000-00001

102. Stamper BD, Park SS, Beyer RP, Bammler TK, Farin FM, Mecham B, et al. Differential expression of extracellular matrix-mediated pathways in singlesuture craniosynostosis. PLoS One. 2011;6(10):e26557. DOI: https://doi.org/10.1371/journal.pone.0026557
2003;111(6):2032-48.

DOI: https://doi.org/10.1097/01.PRS.0000056839.94034.47

104. Penfold JL, Simpson DA. Premature craniosynostosis-a complication of thyroid replacement therapy. J Pediatr. 1975;86(3):360-3. DOI: https://doi.org/10.1016/S0022-3476(75)80963-22

105. Carpenter TO. New perspectives on the biology and treatment of X-linked hypophosphatemic rickets. Pediatr Clin North Am. 1997;44(2):443-66. https://doi.org/10.1016/S0031-3955(05)70485-5

106. Baumgartner JE, Seymour-Dempsey K, Teichgraeber JF, Xia JJ, Waller AL, Gateno J. Nonsynostotic scaphocephaly: the so-called sticky sagittal suture. J Neurosurg. 2004;101(1 Suppl):16-20. DOI: https://doi.org/10.3171/ped.2004.101.2.0016

107. Rowe PSN, Garrett IR, Schwarz PM, Carnes DL, Lafer EM, Mundy GR, et al. Surface plasmon resonance (SPR) confirms that MEPE binds to PHEX via the MEPEASARM motif: a model for impaired mineralization in X-linked rickets (HYP). Bone. 2005;36(1):33-46. DOI: https://doi.org/10.1016/i.bone.2004.09.015

108. Liu J, Kwon T, Nam HK, Hatch NE. Craniosynostosis-Associated Fgfr2 C342Y Mutant Bone Marrow Stromal Cells Exhibit Cell Autonomous Abnormalities in Osteoblast Differentiation and Bone Formation. 2013;ID292506, 11p. DOI: https://doi.org/10.1155/2013/292506

109. Blom HJ, Shaw GM, den Heijer M, Finnell RH. Neural tube defects and folate: case far from closed. Nat Rev Neurosci. 2006;7(9):724-31. DOI: https://doi.org/10.1038/nrn1986

110. Selber J, Reid RR, Chike-Obi CJ, Sutton LN, Zackai $E H, M c D o n a l d-M c G i n n ~ D$, et al. The changing epidemiologic spectrum of single-suture synostoses. Plast Reconstr Surg. 2008;122(2):527-33. DOI: https://doi.org/10.1097/PRS.0b013e31817d548c

111. Clomiphene citrate. Brit Med J. 1968;1(5588):363-4.

Recebido para publicação em 22/08/2016

Revisado em 24/04/2017

Aceito em 14/06/2018

103. Panchal J, Uttchin V. Management of craniosynostosis. Plast Reconstr Surg. Colloq Vitae 2018 mai-ago; 10(2): 65-77. DOI: 10.5747/cv.2018.v10.n2.v234 ISSN 1984-6436/@ 2018 - Publicado pela Universidade do Oeste Paulista.

Artigo Open Access sob uma licença CC BY-NC-ND (http://creativecommons.org/licenses/by-nc-nd/4.0/). 